\title{
Sichere Chirurgie: Operation gelungen! ... und nun?
}

\author{
Anna Mascherek ${ }^{a}$, Paula Bezzolab, Irene Kobler ${ }^{c}$ \\ ${ }^{\text {a } D r ., ~ w i s s e n s c h a f t l i c h e ~ M i t a r b e i t e r i n ~ S t i f t u n g ~ P a t i e n t e n s i c h e r h e i t ~ S c h w e i z ; ~}{ }^{b}$ MPH, Stv. Geschäftsführerin Stiftung Patientensicherheit Schweiz \\ ${ }^{c}$ MA, Projektmanagerin, Stiftung Patientensicherheit Schweiz
}

Als Pilotbetriebe haben mitgemacht: $\mathrm{CHUV}$ Hôpital de l'enfance de Lausanne, freiburger spital / hôpital fribourgeois, Hôpital de la Tour (La Tour Réseau des Soins), Kantonsspital Baden AG (KSB), Kantonsspital Graubünden, Kantonsspital Münsterlingen (Spital Thurgau AG), Kantonsspital Uri, Spitalzentrum Biel AG / Centre hospitalier Bienne SA, Universitätsspital Basel, UniversitätsSpital Zürich mit der Klinik für Angiologie (PTA).
Die chirurgische Checkliste ist ein wichtiges Instrument zur Verbesserung der Patientensicherheit in der Chirurgie. Vor allem bei der Vermeidung schwerer Zwischenfälle spielt sie eine zentrale Rolle. Die korrekte Anwendung und erfolgreiche Implementierung der chirurgischen Checkliste kann nachhaltig jedoch nur gelingen, wenn sie von Begleitmassnahmen flankiert wird, die gesamthaft $A b$ läufe und Prozesse hinterfragen. Um die Patientensicherheit in der Chirurgie substantiell zu verbessern, müssen neben der chirurgischen Checkliste weitere Massnahmen ergriffen werden, welche die Sicherheitskultur im Betrieb positiv beeinflussen. Alle Stakeholder im Gesundheitswesen sind aufgefordert, am gleichen Strick zu ziehen, um die Patientensicherheit in der Chirurgie weiter zu verbessern. Patientensicherheit Schweiz hat hierzu eine Erklärung verfasst, die von namhaften Stakeholdern unterstützt wird.

Das von Patientensicherheit Schweiz durchgeführte 2-jährige Pilotprojekt "progress! Sichere Chirurgie» konnte im Sommer 2015 abgeschlossen werden. Zehn Pilotspitäler ${ }^{1}$ haben die chirurgische Checkliste mit grossem Engagement erfolgreich eingeführt und umgesetzt. Zum Ende des Projekts ist die chirurgische Checkliste in allen Pilotbetrieben in der Routine des OP-Alltags angekommen. Die Checkliste wird standardisiert, personenunabhängig und strukturiert angewendet. Die Erfahrungen haben gezeigt, dass das Erreichen dieses Ziels für alle Beteiligten eine grosse Herausforderung dargestellt hat, die mit viel persönlichem Engagement angenommen und bewältigt wurde. Die Konzeption des Programms, die verpflichtenden Aktivitäten und die interprofessionelle Herangehensweise sind für die erfolgreiche Umsetzung der chirurgischen Checkliste wesentliche Hilfeleistungen gewesen. Als zentrale Aktivitäten für die erfolgreiche Einführung der Checkliste haben sich die lokale Anpassung der Checkliste, die Trainings ausserhalb des OPs sowie die Unterstützung der Führungsebenen erwiesen. Diese Erfahrung wird auch durch die Projektleiter bestätigt. So räumt Dr. med. Antoine Oesch, Leitender Arzt Thoraxchirurgie am Spitalzentrum Biel, ein: «Ohne das Pilotprogramm hätten wir nie so intensive Trainings durchgeführt. Genau die hat es aber gebraucht, um die standardisierte Anwendung mit der notwendigen Interaktion im Team zu erreichen.» Auch die explizite Unterstützung durch die Führungsetage ist in den Pilotbetrieben als wesentlich wahrgenommen worden. Prof. Dr. Jean-Blaise Wasserfallen, ärztlicher Direktor am CHUV in Lausanne, erläutert: «So ein Kulturwandel wird nur möglich, wenn er von erfahrenen Meinungsbildnern angeführt und vorgelebt wird und die Klinikleitung ihn auch mit den nötigen Ressourcen stützt.» Neben dem Engagement und der intensiven Arbeit im Vertiefungsprojekt war die parallel laufende Öffentlichkeitsarbeit wichtiger Bestandteil des Pilotprogramms. Die Kampagne zur Förderung der korrekten Anwendung der chirurgischen Checkliste als professionelle Norm enthielt Elemente auf unterschiedlichen Ebenen wie regelmässig erschienene Fachartikel, Fachreferate, die Publikation wissenschaftlicher Arbeiten sowie verschiedene Medienmitteilungen. Die Pilotbetriebe im Vertiefungsprojekt haben die Aktivitäten im Rahmen der Kampagne mehrheitlich als unterstützend erlebt. So berichtet Tobias Haefliger, Qualitätsbeauftragter am Kantonsspital Uri: «Die Medienmitteilungen zum Projekt fanden ein gutes Echo in der lokalen Presse. Zusätzlich motivierte die Tatsache, dass wir als kleines Pilotspital Pionierarbeit machen, auch die internen Mitarbeitenden.» Diese Erkenntnisse zeigen, dass die Patientensicherheit in der Chirurgie als wichtiges Thema auf die Agenda gesetzt und öffentlichkeitswirksam aufgearbeitet werden konnte. Durch das Engagement von Fachpersonen innerhalb des Vertiefungsprojekts und durch Personen, die unabhängig vom Vertiefungsprojekt mit den Empfehlungen und Unterstützungsmaterialien eigene Verbesserungsprojekte umgesetzt haben, konnte die chirurgische Checkliste in vielen Betrieben erfolgreich in den OP-Alltag integriert werden. Auch das Engagement, das bereits vor Beginn des Pilotprojekts bei Fachpersonen vorhanden war, konnte im Rahmen von "progress! Sichere Chirurgie» weiter zielführend gebündelt werden. Die Schweiz steht heute an einem anderen Ort als zu 


\section{Weiterführendes Angebot «progress! Sichere Chirurgie»}

Neben der Erklärung werden nach Abschluss des Vertiefungsprojekts die Erkenntnisse aufgearbeitet und öffentlich zur Verfügung gestellt. So sollen Spitäler, die nicht am Vertiefungsprojekt teilgenommen haben, unterstützt werden, die chirurgische Checkliste erfolgreich im eigenen Betrieb zu implementieren oder deren Anwendung zu verbessern. Das Material beruht auf Erkenntnissen aus der Literatur, auf internationalem und nationalem Expertenwissen, auf Modellen zur Entwicklung von Verbesserungsprogrammen und auf den im Vertiefungsprojekt gemachten Erfahrungen. Die Schlüsselaspekte und zentralen Aktivitäten, die massgeblich zum Erfolg beigetragen haben, sind dargestellt und werden als Vorlagen und Beispiele zur Verfügung gestellt. Die Unterlagen können unter www. patientensicherheit.ch bezogen werden. Ein E-Learning-Kurs zur Wissensvermittlung kann zudem käuflich erworben werden.

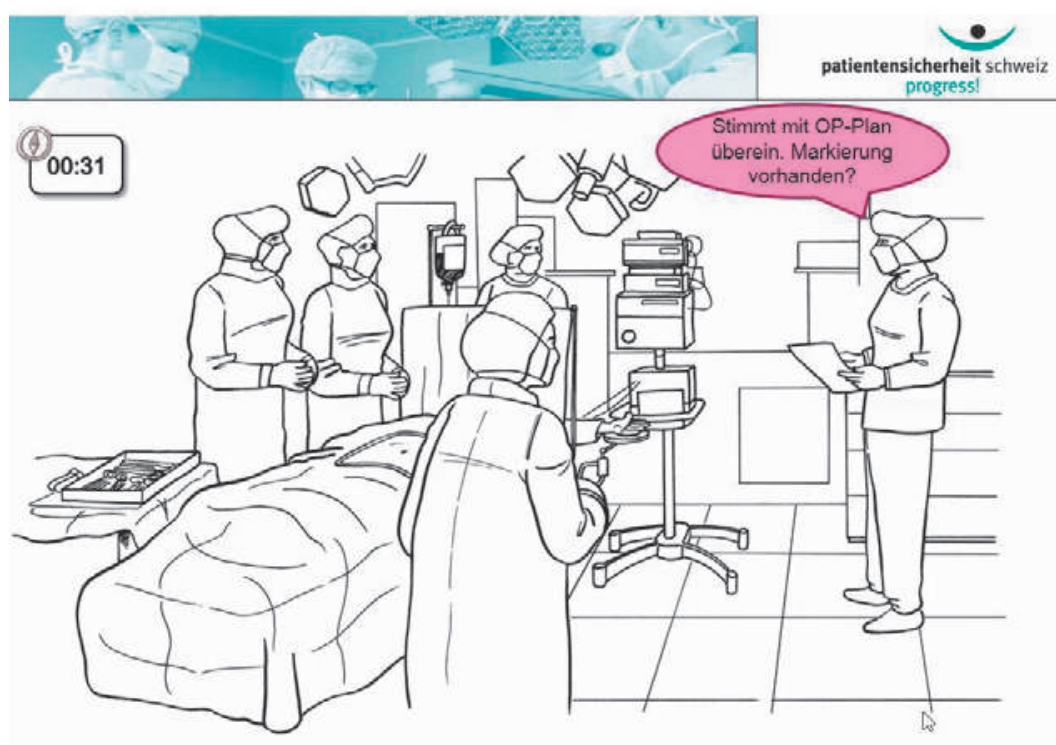

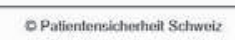

2 Weitere Informationen zur Umfrage erhalten Sie auf Anfrage bei Patientensicherheit Schweiz.
Beginn des Projekts im Sommer 2013 (siehe auch [1] zur Ausgangslage in der Schweiz). Die Anwendung der Checkliste ist stärker im Berufsalltag verankert und wird mehr und mehr zur professionellen Norm. Dies zeigen auch die Resultate einer nicht-repräsentativen Online-Befragung von Patientensicherheit Schweiz bei insgesamt 1194 invasiv tätigen Ärzten und Ärztinnen, Anästhesiepersonal und leitendem OP-Personal aus dem Frühling 2015. So zeigt sich in der subjektiven Wahrnehmung der Befragten eine höhere Präsenz des Themas Patientensicherheit in der Chirurgie: Knapp $85 \%$ der Befragten geben an, dass ihrer Meinung nach heute mehr für die Patientensicherheit getan wird als noch vor einem Jahr. Bei der Interpretation der Resultate muss berücksichtig werden, dass Personen, die in einem Pilotbetrieb gearbeitet haben, in der Stichprobe überrepräsentiert sind. Die Ergebnisse zeigen aber insgesamt, dass die Checkliste als wichtiges Thema im Alltag angekommen ist ${ }^{2}$. Es zeigt sich, dass die Checkliste einen wichtigen Beitrag zur Verbesserung der Patientensicherheit leisten kann, wenn sie sorgfältig und mit viel Engagement durch die Fachpersonen an der Basis implementiert wird. Im Projekt wurde aber auch deutlich, dass die Checkliste kein simples Tool zum Abhaken ist, sondern am Ende eines gesamthaften Prozesses zur Verbesserung der Patientensicherheit in der Chirurgie steht. Für die effiziente und wirksame Anwendung braucht es eine Reihe von vorgelagerten Schritten. Sie kann nachhaltig nur gelingen, wenn sie von Massnahmen begleitet wird, die gesamthaft Abläufe und Prozesse im OP hinterfragen.

Die richtige Anwendung der Checkliste stellt eine komplexe Teamintervention dar, welche die gesamte Kultur einer chirurgischen Abteilung betrifft und die Teamkommunikation und -zusammenarbeit verändern kann. Sie erfordert, dass vor- und nachgelagerte Prozesse sowie Routinen hinterfragt und angepasst werden. Rollen und Verantwortungen müssen klar definiert werden. Die Einführung der Checkliste ist insofern als "Aufhänger» zu betrachten, um die Patientensicherheit in der Chirurgie substantiell und nachhaltig auf unterschiedlichen Ebenen zu thematisieren. Dies ist auch bei der Implementierung der Checkliste im Pilotprogramm deutlich geworden. Dr. med. Marianna Friedli-Braun, Leitende Ärztin Chirurgie am Kantonsspital Graubünden, kann aus ihrer Erfahrung bestätigen: «Um die Zufriedenheit und Compliance zu erhöhen, war für uns die lokale Anpassung der Checkliste zentral. Konkret haben wir z.B. das Sign Out ergänzt, das Format und Layout angepasst, die Antwortfelder klarer gestaltet und fixe Zuständigkeiten definiert. Das alles soll den Ablauf klarer und sicherer machen.» Die Definition von Zuständigkeiten, das Anpassen der Checkliste auf lokale Gegebenheiten, das Hinterfragen von Routinen sind also notwendige Vorarbeiten, an deren Ende erst die Anwendung der Checkliste als Tool steht.

\section{Checkliste als erster Schritt in Richtung einer verbesserten Sicherheitskultur}

Die Anwendung der chirurgischen Checkliste stellt nur eine notwendige Massnahme unter vielen dar, um die Patientensicherheit in der Chirurgie zu erhöhen. Um im Betrieb die Patientensicherheit als Ganzes betrachten zu können und Verbesserungen nachhaltig auf allen Ebenen spürbar zu machen, können ergänzende sicherheitswirksame Massnahmen schrittweise umgesetzt werden. Das Sicherheitsklima als umgebender, unspezifischer Faktor in einem Betrieb kann die erfolgreiche Umsetzung von Massnahmen begünstigen oder erschweren und muss dementsprechend berück- 
sichtigt werden [2, 3]. Je nachdem, inwieweit der konstruktive Umgang mit Fehlern, das Ernstnehmen anderer Professionen und das sachliche Zusammenarbeiten unabhängig von Hierarchien bereits gepflegt werden, muss die Verbesserung der Sicherheitskultur als explizites Ziel definiert und angegangen werden. Generelle Massnahmen zur Verbesserung der Sicherheitskultur können beispielsweise die Verbesserung der interprofessionellen/interdisziplinären Zusammenarbeit und der Kommunikation sein. Hierfür können Teamtrainings wie Crew Ressource Management (CRM), Simulationstrainings oder strukturierte Übergabeprotokolle herangezogen werden.

Auch konkrete Projekte in anderen Bereichen als der chirurgischen Checkliste können dazu beitragen, die Patientensicherheit zu verbessern. So sind Massnahmen zur Verbesserung der Arbeitsumgebung, wie zum Beispiel die Reduktion des Lärmpegels oder das Schaffen geeigneter Lichtverhältnisse, ebenfalls wichtige, wenn auch "pragmatische» Aspekte. Bei allem Bemühen, Fehler zu reduzieren und zu vermeiden, ist eine komplette Fehlerfreiheit auch in bestens aufgestellten Betrieben nicht zu erreichen. Wo viele Menschen in komplexen Umwelten arbeiten, passieren Fehler. Die Fehler, die dann zumeist erst am Patienten zutage treten, haben sich aber in der Regel schon viel früher auf systemischer Ebene angebahnt [4]. Aus diesem Grund sind beispielsweise systemische Fehleranalysen oder Mortalitäts- und Morbiditätskonferenzen wesentliche Massnahmen, die zur Verbesserung der Sicherheit in einem Betrieb beitragen können. Wenn Fehler angemessen aufgearbeitet und Konsequenzen daraus gezogen werden, kann ein Betrieb als lernende Organisation beständig daran arbeiten, die Sicherheit im eigenen System zu verbessern und ein gutes Sicherheitsklima zu fördern.

\section{Beitrag der Stakeholder}

Um die obengenannten Aspekte umsetzen und so nachhaltig die Patientensicherheit in der Chirurgie verbessern zu können, brauchen die Betriebe einen Rahmen, in dem sie Veränderungen und Ressourcen aufbieten und verankern können. Die Einführung der Checkliste ist mit so deutlichen Veränderungen vor Ort verbunden, dass die mandatorische Einführung per Vorgabe auf politischer Ebene allein nicht zielführend ist. Vorgaben müssen auch die Rahmenbedingungen berücksichtigen, die für eine wirkungsvolle Umsetzung notwendig sind. Deshalb müssen Stakeholder und Fachpersonen aus der Politik, in der Aus- und Weiterbildung und in den Betrieben auf allen Ebenen in ihrem jeweiligen Wirkungsrahmen die korrekte Anwendung der Checkliste bei allen Patienten unterstützen. Patientensicherheit ist eine Aufgabe, die in allen Berufsgruppen als professionelle Norm verankert sein muss. Zusätzlich muss das Thema speziell auf Führungsebene in den verschiedenen Berufsgruppen im Gesundheitswesen einen Platz haben. Die Stiftung Patientensicherheit Schweiz fordert deswegen alle Stakeholder im Gesundheitswesen auf, auf der Basis der Erkenntnisse aus dem Vertiefungsprojekt Grundlagen zu schaffen, auf die sich die Betriebe berufen können, wenn sie Massnahmen zur Verbesserung der $\mathrm{Pa}$ tientensicherheit in der Chirurgie ergreifen. Auch ohne gesetzliche Grundlage zur Einführung der Checkliste müssen wichtige Stakeholder im Gesundheitswesen, d.h. Personen, Gremien und Organisationen, die eine Führungs- oder Ausbildungsfunktion innerhalb des Gesundheitswesens innehaben, die Einführung der Checkliste und die damit verbundenen Analysen und Anpassungen von bestehenden Prozessen explizit unterstützen. Die verschiedenen Stakeholder im Gesundheitswesen können im Rahmen ihrer Funktionen und ihrer Aufträge Massnahmen zur Verbesserung der Patientensicherheit fördern. Ein erster Schritt in diese Richtung ist bereits erfolgt: Im November 2015 hat Patientensicherheit Schweiz an einer Sitzung mit verschiedenen Stakeholdern wichtige Aspekte zur Patientensicherheit in der Chirurgie diskutiert. Als Ergebnis dieser Sitzung treten die unterschiedlichen Stakeholder als Befürworter einer gemeinsamen Erklärung zur Verbesserung der Patientensicherheit in der Chirurgie und damit verbundenen notwendigen Massnahmen auf. Sie verpflichten sich, die in der Erklärung formulierten Erkenntnisse und Empfehlungen in die Aktivitäten und Bemühungen ihrer Organisationen zu integrieren bzw. dort zu berücksichtigen. Die Erklärung im Wortlaut mit allen unterstützenden Stakeholdern ist unter www.patientensicherheit.ch verfügbar. Weitere Organisationen und Einzelpersonen sind eingeladen, die Erklärung via info[at]patientensicherheit.ch zu unterzeichnen.

\section{Literatur}

1 Mascherek A, Schwappach D, Bezzola P. Frequency of use and knowledge of the WHO-surgical checklist in Swiss hospitals: a cross-sectional online survey. Patient safety in surgery. $2013 \cdot 7(1) \cdot 36$

2 McFadden KL, Henegan SC, Gowen III CR. The patient safety chain: Transformational leadership's effect on patient safety culture, initiatives, and outcomes. Journal of Operations Ma nagement. 2009;27(5):390-404.

3 Mardon RE, Khanna K, Sorra J, Dyer N, Famolaro T. Exploring Relationships Between Hospital Patient Safety Culture and Adverse Events. Journal of Patient Safety. 2010;6(4).

4 Reason J. Human error: models and management. BMJ. 2000;320(7237):768-70. 